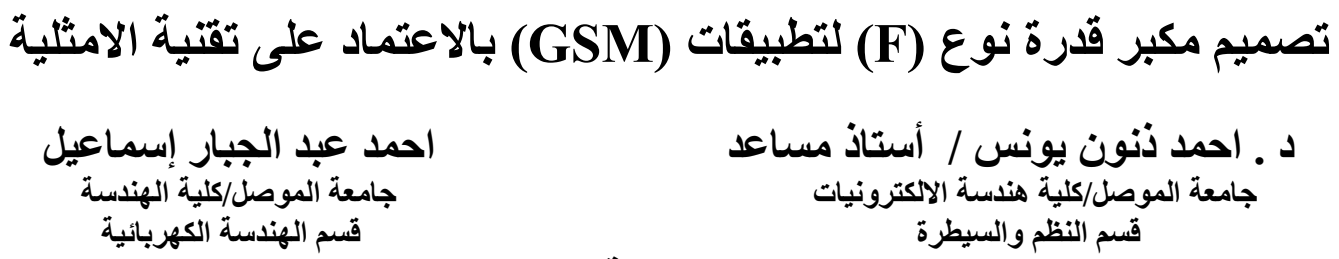

\title{
الخلاصة
}

مكبر القدرة نوع (F) هو احد أنواع مكبرات القدرة المفتاحية الأكثر استخداما في أنظمة الاتصالات الراديوية

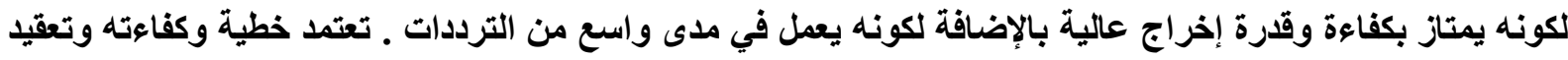

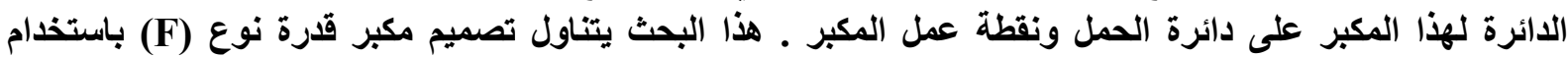

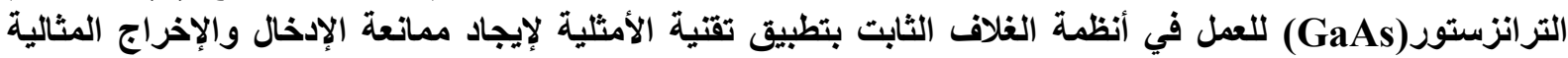

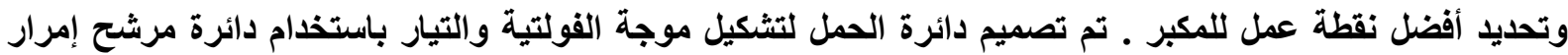

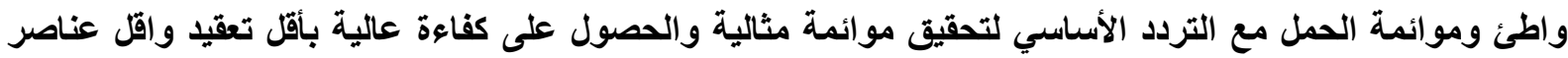

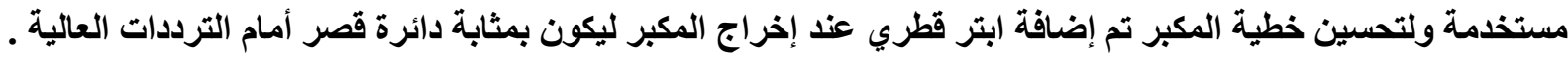

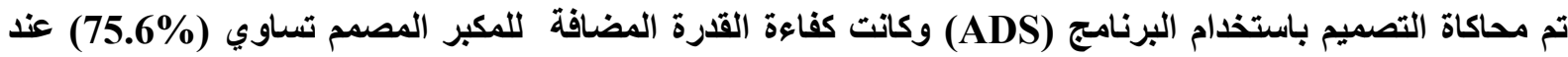

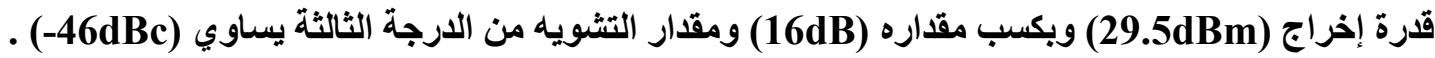

الكلمات الدالة: مكبرات القدرة المفتاحية ، دائرة الحمل ، تثكيل الموجة ، أنظمة (GSM)

\section{Design Class (F) Power Amplifier for (GSM) Application Based on Optimization}

\author{
Ahmad T. Younis \\ University of Mosul \\ Electronic Engineering College \\ Systems and Control Dept.
}

\author{
Ahmad Abdul-Jabbar Ismail \\ University of Mosul \\ Electrical Dept.
}

\section{Abstract}

Non-linear Class-F PA has drawn a great attention among all different classes of PAs because of their capability of giving high power, providing good PAE and work in high frequency .The problem of class F PA is Poor linearity. The efficiency linearity and complexity of class (F) power amplifier depend on the load network. In this research, Class-F amplifier with carefully chosen bias points, input and output impedance, designed load matching network and harmonic traps to get linearity and efficiency greeting with (GSM) application . The load network was designed by low pass flitter and matching network was designed to obtain the required optimum impedance at fundamental only to reduce complexity of $\mathrm{PA}$ and adding radial stub to provide a short circuit for higher degree harmonics and improve the linearity. The final design produced a PAE of $75.6 \%$ with $29.5 \mathrm{dBm}$ output power with $(16 \mathrm{~dB})$ gain and $\mathrm{TH}$ distorted $-46 \mathrm{dBc}$. 


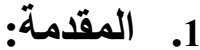

تعد الكفاءة العالية لمكبر القدرة من الأمور المهمة جدا في أجهزة الاتصال الحديثة وبخاصة في محطات الإرسال وذللك لتقليل استهلاك القدرة وتقليل الكلفة وتصغير الحجم ـ لذلك يتم بناء مكبرات القدرة باستخدام مكبر ات القدرة المفتاحية

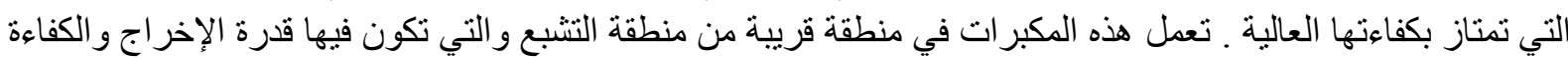

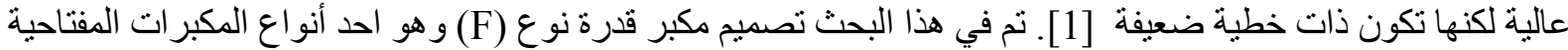

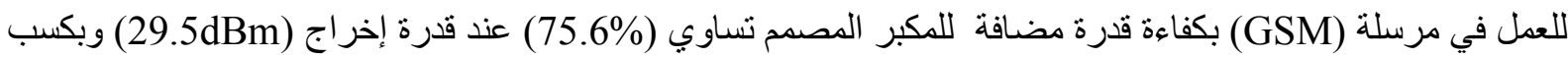

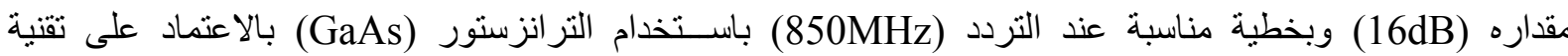

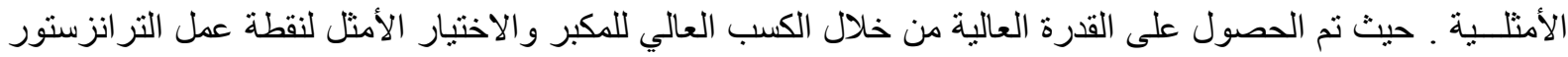

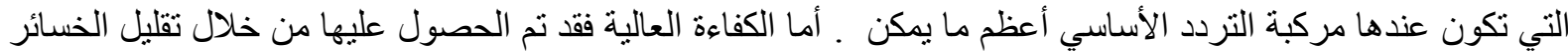

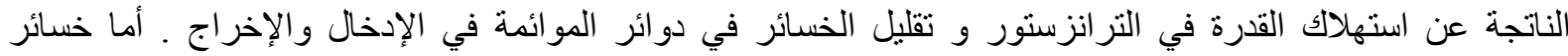

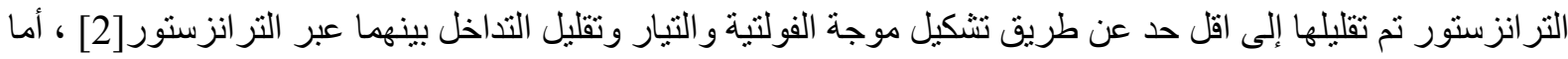

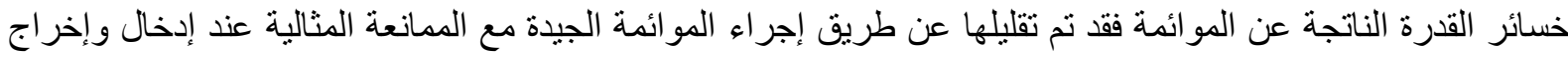

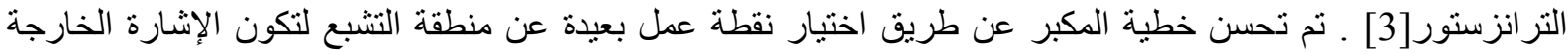

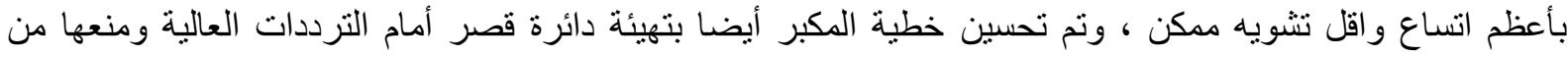
الوصول للحمل باستخدام ابتر قطري (radial stub) الذي يعمل كمرشح و إمرار و اطنئ .

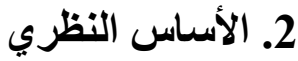

\section{1. علاقة الكفاءة وقدرة الإخراج بزاوية التوصيل}

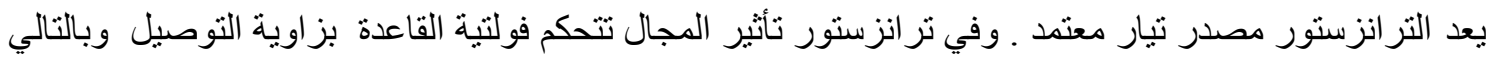

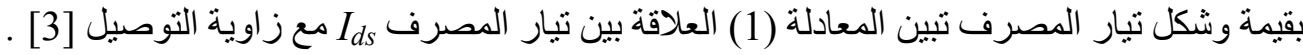

$\boldsymbol{i}_{d s}\left(\theta_{)}=I_{\max } * \frac{\cos \theta-\cos \left(\alpha \alpha^{\prime} 2\right)}{1-\cos (\alpha / 2)}\right.$

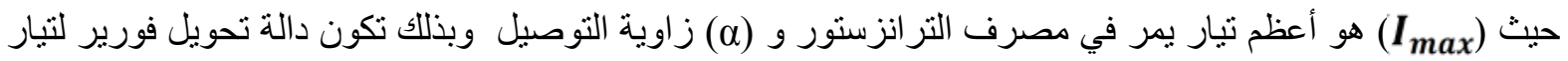

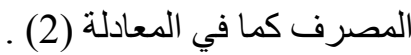

$i_{d s}(\theta)=I_{d c}+I_{1} \cos \theta+I_{2} \cos 2 \theta+I_{3} \cos 3 \theta+\cdots$

وتكون قيم المركبات الثلاث الأولى وقيمة مركبة التبار المستمر بدلالة كما في المعادلة (3) .

$I_{d C}=\frac{I_{\max }}{2 \pi} * \frac{2 \sin \left(\alpha^{\prime} 2\right)-\alpha \cos \left(\alpha^{\prime} 2\right)}{1-\cos \left(\alpha^{\prime} 2\right)}$

$I_{1}=\frac{I_{\max }}{2 \pi} * \frac{\alpha-\sin \alpha}{1-\cos \left(\alpha^{\prime} 2\right)}$

$I_{2}=\frac{I_{\max }}{2 \pi} * \frac{2 \sin \left(\alpha^{\prime} 2\right)-\frac{1}{3} \sin \left(3 \alpha^{\prime} / 2\right)}{1-\cos \left(\alpha^{\prime} 2\right)}$

$I_{3}=\frac{I_{\max }}{6 \pi} * \frac{(1-\cos \alpha) \sin \alpha}{1-\cos \left(\alpha^{\prime} 2\right)}$

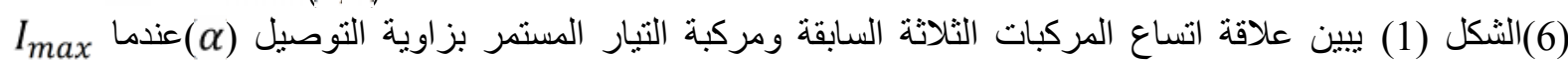

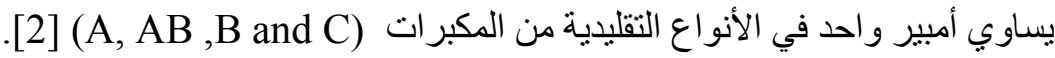


العلاقات السابقة تبين إن شكل موجة

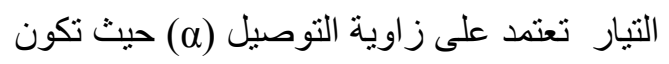

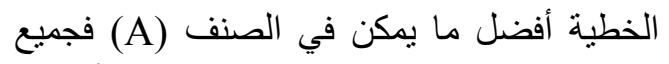

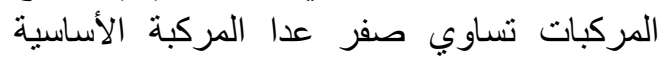

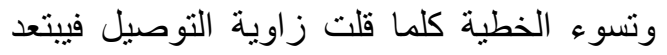

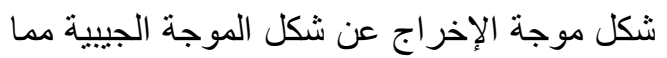

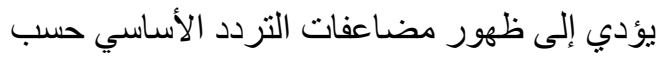

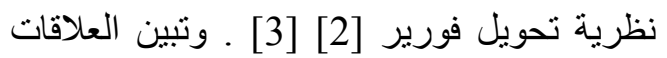

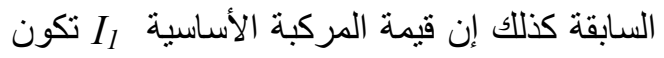

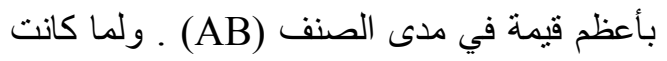
كفاءة المكبر تعتمد على قدرة الإخراج والقدة القدرة

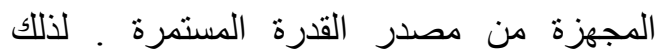

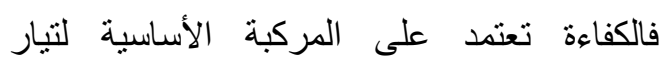

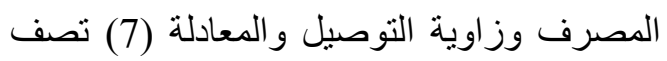

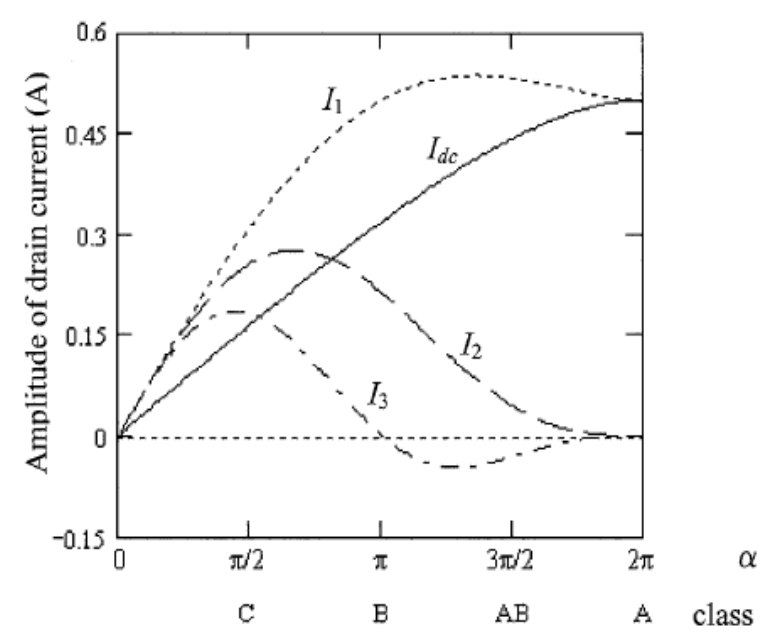

$I_{m a x}=1 \mathrm{~A}$ الثكل (1) تغير اتساع مركبات التيار عندما يكون

$$
\eta_{d}=\frac{P_{o u t}}{P_{d c}}=\frac{1}{2}\left(\frac{\alpha-\sin \alpha}{2 \sin \left(\alpha^{\prime} 2\right)-\alpha \cos \left(\alpha^{\prime} 2\right)}\right)
$$

يمكن بناء مكبر القدرة (F) من الأصناف التقليدية من المكبرات(B,AB,C) [5 [B] وقد تم في هذا البحث اختيار نقطة

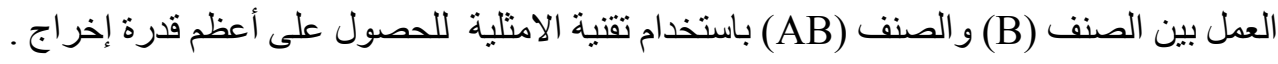

2.2. - 2. 2

إن دائرة مكبر القدرة من الدو ائر غير الخطية يمكن تمثيلها على أنها دائرة ذات منفذين يمكن تمثيل خصائصها بعدة

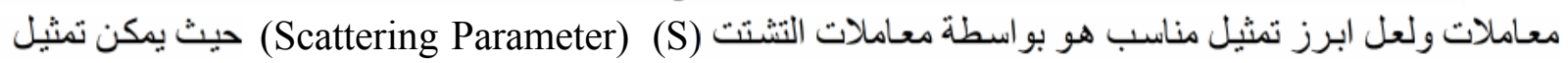

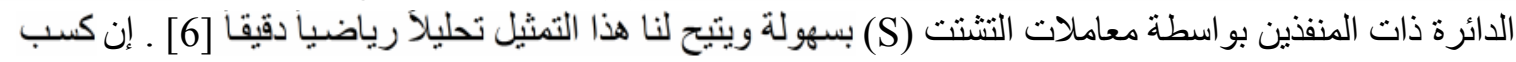
(Ga)

(GT) القدرة بعتمد بصورة أساسية :[8],[7] (Gp)

$$
\begin{aligned}
& G_{T}=\frac{1-\left|\Gamma_{S}\right|^{2}}{\left|1-\Gamma_{i n} \Gamma_{S}\right|^{2}}\left|S_{21}\right|^{2} \frac{1-\left|\Gamma_{L}\right|^{2}}{\left|1-S_{22} \Gamma_{L}\right|^{2}} \\
& G_{a}=\frac{1-\left|\Gamma_{S}\right|^{2}}{\left|1-S_{11} \Gamma_{S}\right|^{2}}\left|S_{21}\right|^{2} \frac{1}{1-\left|\Gamma_{\text {out }}\right|^{2}} \\
& \mathrm{C}_{\mathrm{P}}=\frac{1}{1-\left|\Gamma_{\text {in }}\right|^{2}}\left|\mathrm{~S}_{21}\right|^{2} \frac{1-\left|\Gamma_{L}\right|^{2}}{\left|1-\mathrm{S}_{22} \Gamma_{L}\right|^{2}}
\end{aligned}
$$$$
\text { هـي }
$$

(transducer power gain) (available power gain)

عندما تكون المو ائمة جيدة في الإدخال و الإخر اج فان معاملات الانعكاس و التشتت

$\Gamma_{\text {in }}=S_{11}, \quad \Gamma_{\text {out }}=S_{22}, \Gamma_{G}=\Gamma_{L}=0$

و عند هذه الحالة يمكن اختز ال معاملات الكسب إلى:

$G_{T}=\left|S_{21}\right|^{2}, G_{a}=\frac{\left|S_{21}\right|^{2}}{1-\left|S_{22}\right|^{2}} \quad, G_{P}=\frac{\left|S_{21}\right|^{2}}{1-\left|S_{11}\right|^{2}}$

بدرجة الموائمة في الإدخال و الإخر اج هي العلاقات التالية [7][8]: 
$M_{i n}=\frac{P_{i n}}{\mathrm{P}_{\mathrm{AVS}}}=\frac{G_{T}}{G_{P}}=\frac{\left(1-\left|\Gamma_{i n}\right|^{2}\right)\left(1-\left|\Gamma_{S}\right|^{2}\right)}{\left|1-\Gamma_{i n} \Gamma_{S}\right|^{2}}$

$M_{\text {out }}=\frac{P_{L}}{P_{A V N}}=\frac{G_{T}}{G_{a}}=\frac{\left(1-\left|\Gamma_{\text {out }}\right|^{2}\right)\left(1-\left|\Gamma_{L}\right|^{2}\right)}{\left|1-\Gamma_{\text {out }} \Gamma_{L}\right|^{2}}$

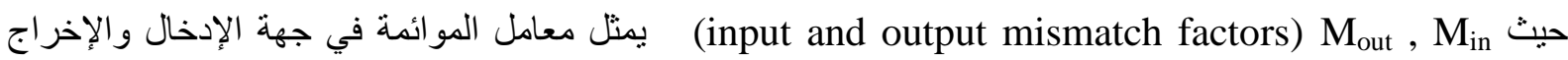
(16,15)

فتصبح بالصيغة التالية : (dB) و أفضل قيمة لهما تساوي واحد (unity) عندها يكون ) ع (8) فهو يتأثر بمو ائمة الإدخال و الإخر اج ـ ويمكن التعبير عن العادلة (15)

$\left.M_{i n}\right|_{d B}=\left.G_{P}\right|_{d B}-\left.G_{T}\right|_{d B}$

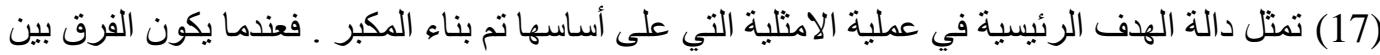

الكسب الإجمالي والكسب المؤثر قليل وقريب من الصفر فهذا يعني أن الموائمة جيدة والمكبر يعمل في المنطقة الخطية (17) $\left(S_{21}\right)$ و عندها تصبح قيمة الكسب الإجمالي والكبالي الكوبر ـ وبحسب متطلبات التصميم يمكن كتابة المعادلة (17) بالصيغة التالية .

$G_{P \mid d B}-G_{T \mid d B}<1 d B$

(18) هي تحوير من المعادلة (17) بعد الاخذ بنظر الاعتبار الثروط الحدية المطلوبة لعملية الامثلية

لتحقيق افضل و اقل قيمة لعامل المو ائمة في الادخال و ادخلت كمعادلة رئيسية في خو ارزمية الامثلية .

3. تركيب مكبر القدرة

3.1.تصميم دائرة تشكيل الموجة مئرة

ان النقطة الاساسية في كفاءة مكبرات القدرة المفتاحية هي هندسة تشكيل الموجة والتي يتم من خلالها تقليل التداخل

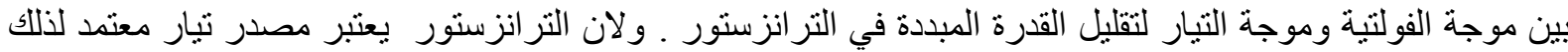

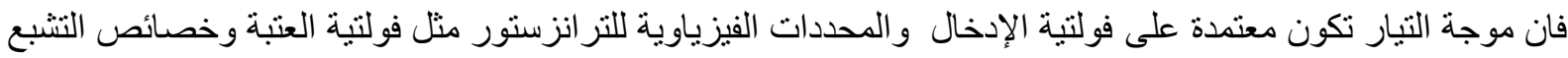
و العناصر الطفيلية ـ و هكذا تكون موجة التيار حاملة لعدد غير منتهي من التوارفيات الإديات كما في المعادلة (2) . يتم تشكيل تيار المصرف بمان فيئة موجة (F)

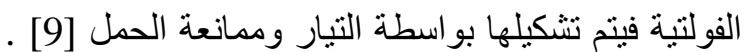

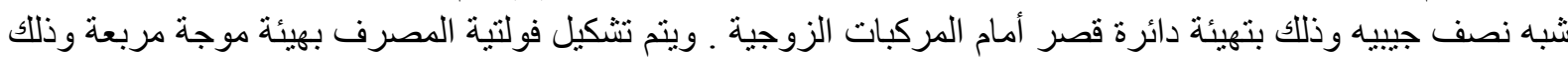

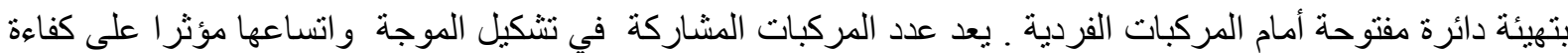
(F)

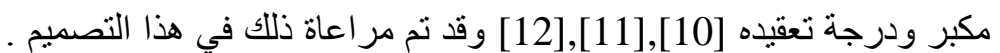

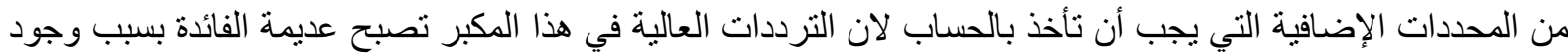

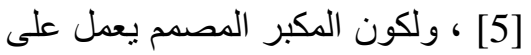
(parasitic) العناصر الطفيلية (850)

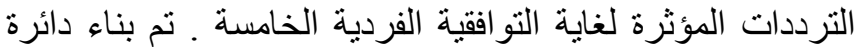
ترددات عالية (850 MHz)

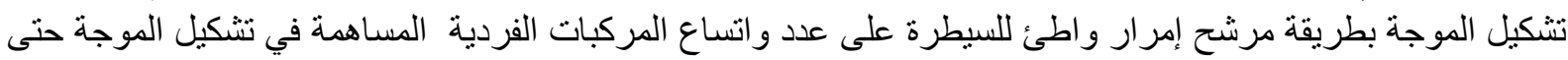
المركي

المركبات المختلفة للحصول اتساع مناسب لتلك المركبة عند إخراج الترانزستور وتم تتفيذه باستخدام مرشح نوع والذي يحقق المو اصفات التالية : (Filter order =5) (2) يبين دائرة الـ الـانبان (Chebyshev Filter) ( Stop band Frequency (Fs) =3.315 GHz ) (Pass band Corner Frequency $(\mathrm{Fp})=2.55 \mathrm{GHz}$ ) . ( Stop band Attenuation $(\mathrm{As})=20 \mathrm{~dB}) \quad($ Pass band ripple $(\mathrm{Ap})=3$ ) 


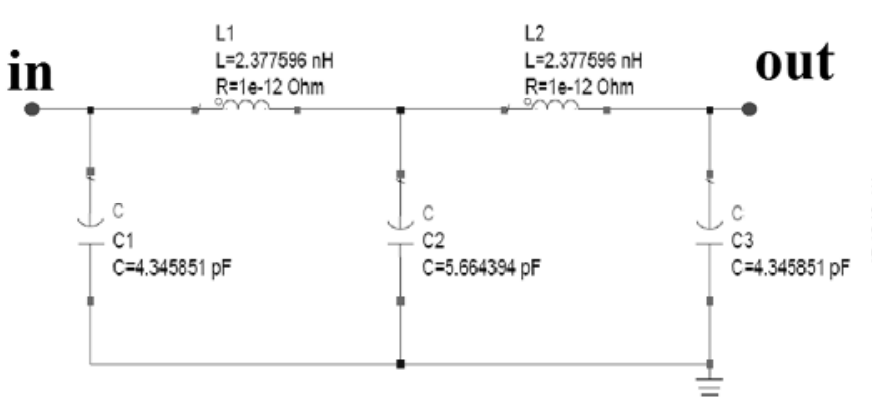

(a)

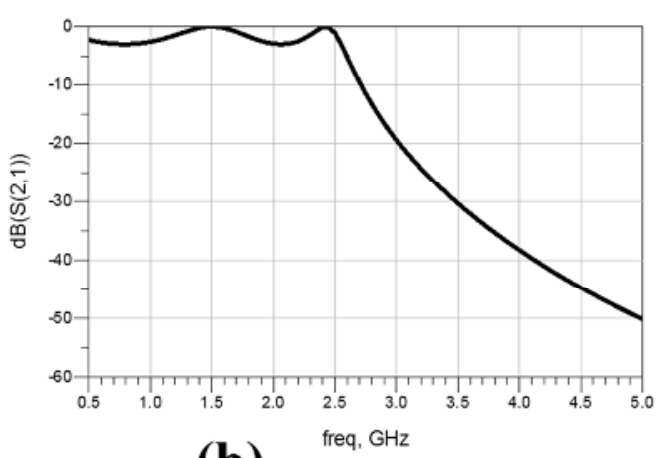

(b)

والثكل (2) : (a) دائرة مرشح امرار واطئ (b) الاستجابة الترددية لعامل الكسب مع التردد

أما المركبات الزوجية فيتم السيطرة عليها من خلال دائرة القصر التي يوفر ها خط النقل (2/4)

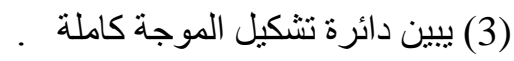
. (C PASS

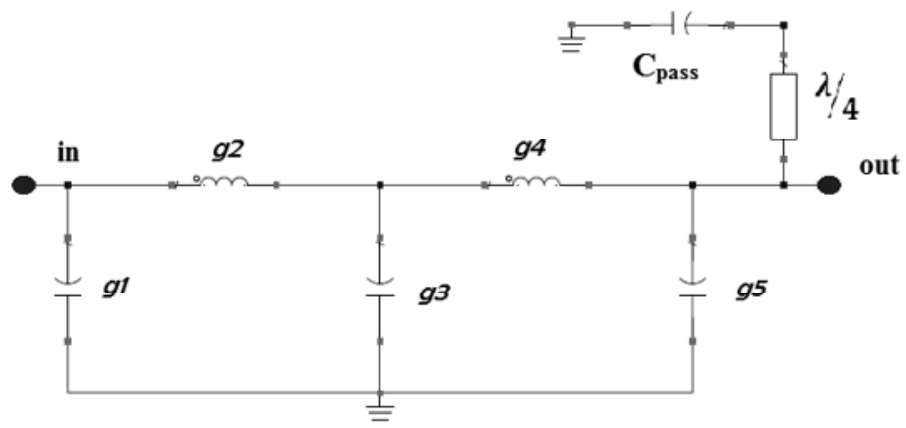

(3) الثكل (3) دائرة تثكيل الموجة لمكبر القرة نوع (F)

\section{2. إيجاد ممانعة الإدخال والإخراج بطريقة الامثلية}

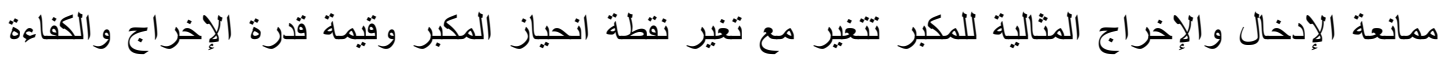

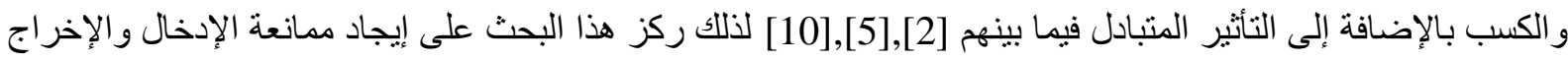

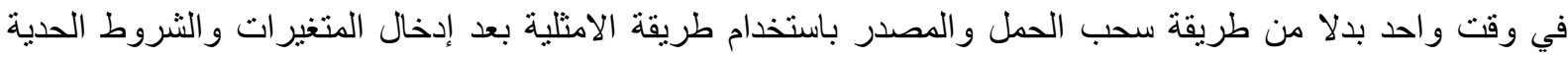

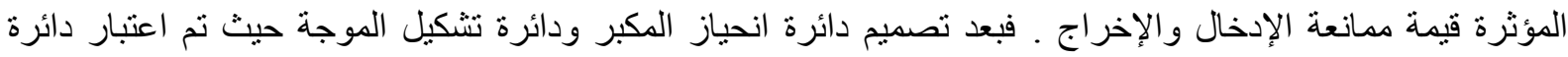

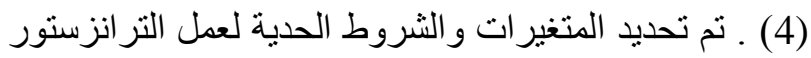

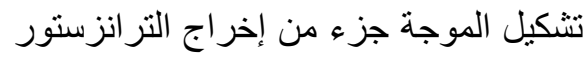

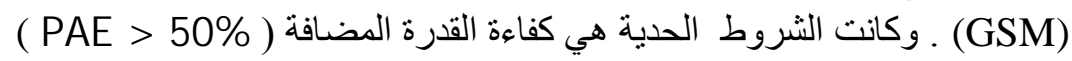

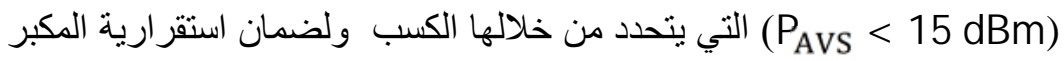
$\left(P_{L}>26 \mathrm{dBm}\right)$

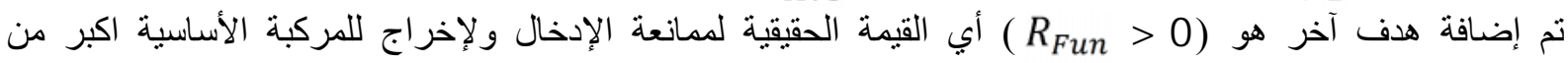

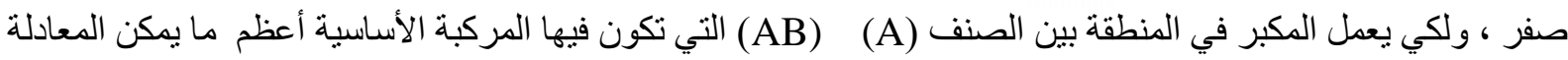

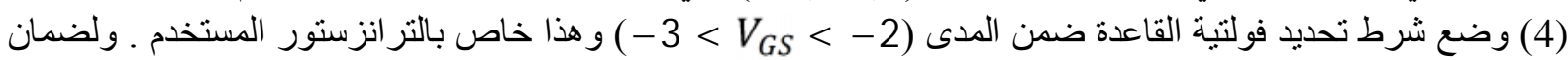

$$
\text { المكبر في المنطقة الخطية ونقل أعظم قدرة }
$$

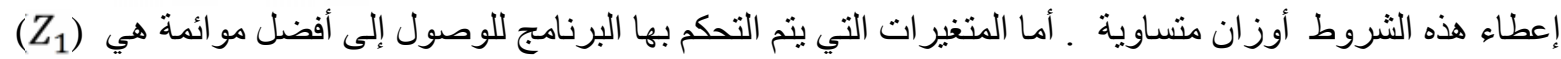

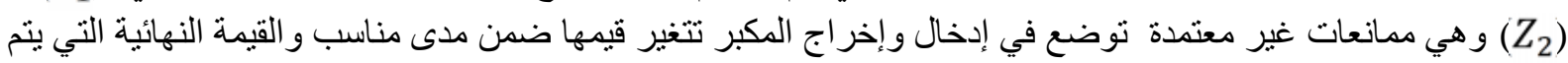


عندها تحقيق الأهداف هي الد انعة المثالية وهي دالة الهدف وتم تنفيذ عملية الامثلية باستخدام خوارزمية كر ادين (ADS)

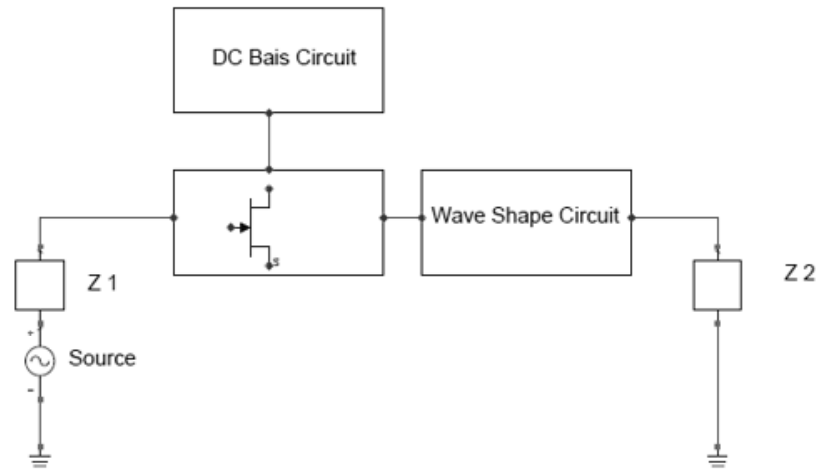

الثكل (4) مخطط عام يبين طريقة إيجاد ممانعة الإدخال والإخراج المثالية للمكبر

4. تحقيق وتركيب مكبر القدرة

بعد أيجاد قيمة الممانعة المثالية في الإخخال والإخر اج وتصميم دوائر الموائمسة تم بنـاء المكبر بطريقة العناصر

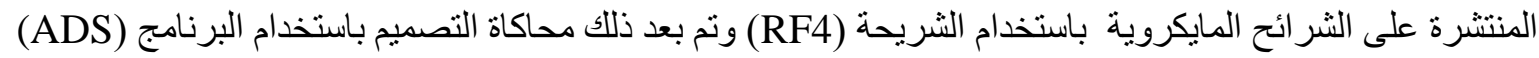

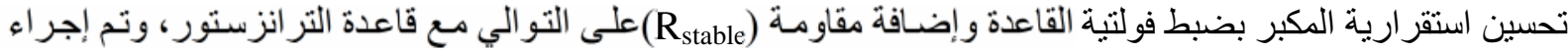

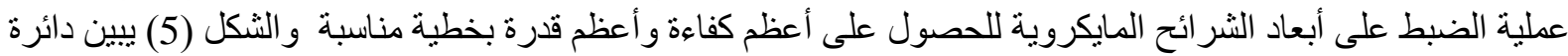

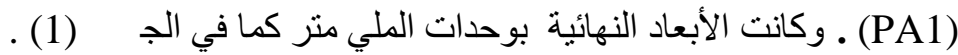

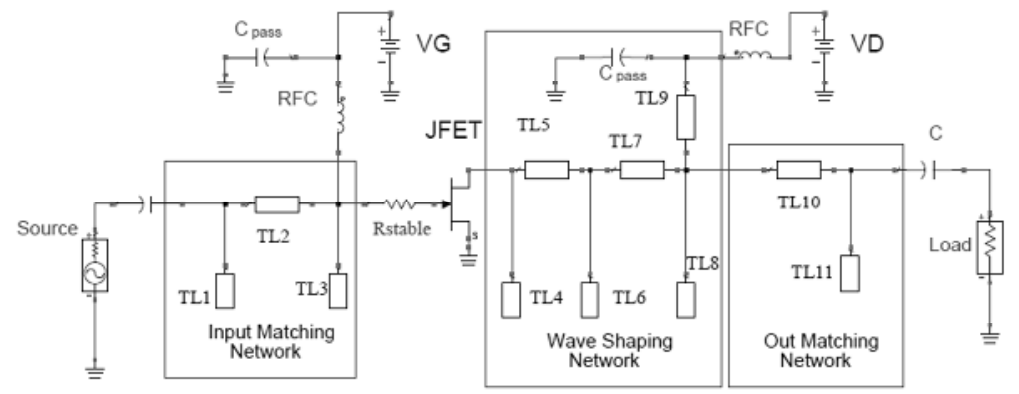

الشكل (5) دائرة مكبر القدرة نوع (FA1) الدائرة الاساسية (PA)

جدول (1) أبعاد خطوط النقل بعد إجراء عملية الضبط بالملي متر

\begin{tabular}{|l|c|r|r|r|r|r|r|r|r|r|r|}
\hline & TL1 & TL2 & TL3 & TL4 & TL5 & TL6 & TL7 & TL8 & TL9 & TL10 & TL11 \\
\hline $\mathrm{W}$ & 3.088 & 3.093 & 3.088 & 3.088 & 3.093 & 3.088 & 3.09 & 3.088 & 3.088 & 3.088 & 3.088 \\
\hline L & 37.4 & 22.18 & 29 & 26.5 & 7.8 & 30.64 & 7.84 & 48.8 & 26.5 & 16.54 & 15.45 \\
\hline
\end{tabular}

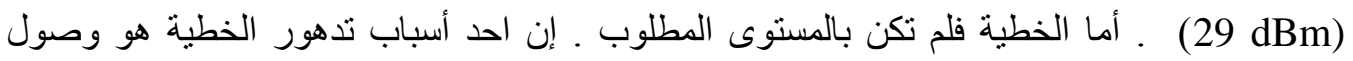
التو افقيات إلى الحمل بسبب عدم توفر دائرة قصر مثالية عن طريق خط النقل (4/2) (TL9) عبر منسعة التسريب 
عند نهاية خط النقل (Radial stub)

PA1 إلى الأرضي ، تم تحوير دائرة المكبر (C C

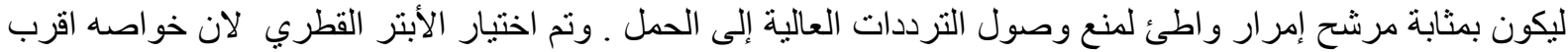

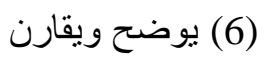

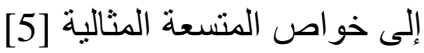

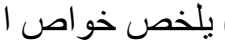
ذو النهاية المفتوحة

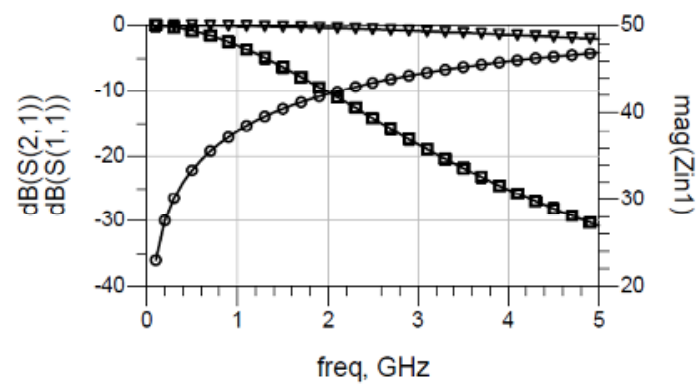

(a)

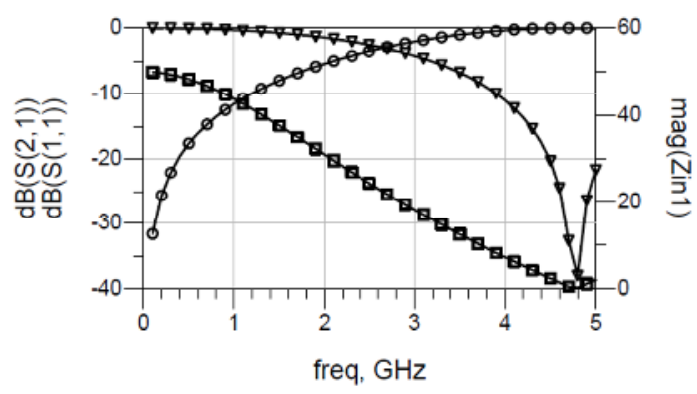

(b)

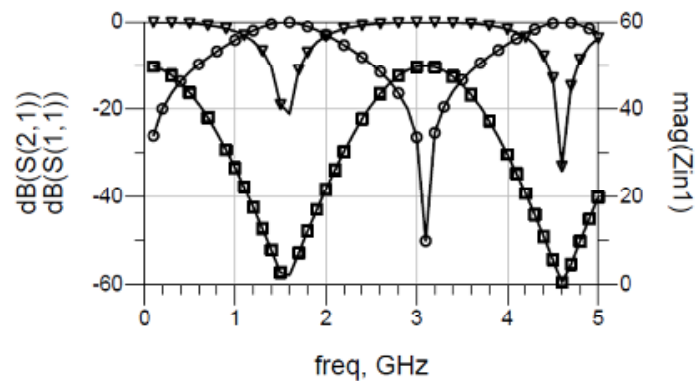

(c)

$\operatorname{mag}($ Zin1 $) \triangle \quad \mathrm{dB}(\mathrm{S}(2,1)) \quad \square \quad \mathrm{dB}(\mathrm{S}(1,1)) \quad 0$

الثكل(6): مقارنة الممانعة والتوهين لكل من (a) المتسعة (b)خواص الأبتر القطري (c) الأبتر ذو النهاية المفتوحة المعة

الجدول (2) يلخص خواص الأبتر القطري مقارنة بخواص المتسعة والأبتر ذو النهاية المفتوحة

\begin{tabular}{|c|c|c|c|}
\hline & 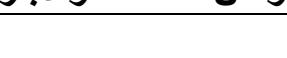 & المثالية & \\
\hline ضيق & عريض & عريض & مدى الممانعة الصغيرة \\
\hline \multicolumn{4}{|l|}{ 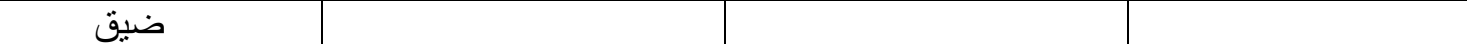 } \\
\hline يستجيب للتو افقيات & لا يستجيب للتو افقبات & لا يستجيب للتو افقيات & استجابته للتو افقيات \\
\hline
\end{tabular}

(7) كانت على حساب الكفاءة حيث قلت الكفاءة

إن تحسين الخطية بإضافة الإبر القطري(PA2)

$(70 \%)$ 


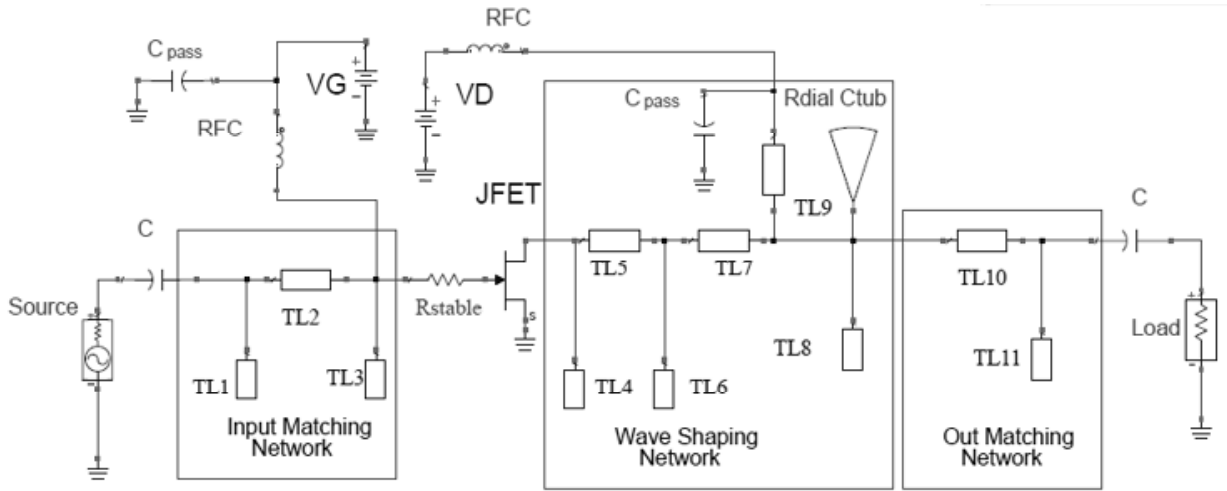

الثكل (7) تركيب دائرة مكبر القدرة صنف PA2 (F)

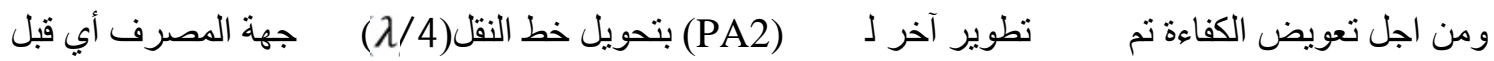
PA3 (8)

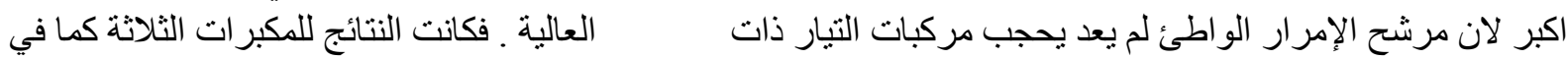

\begin{tabular}{|c|c|c|c|}
\hline & PA1 Fig.(3.20) & PA2 Fig.(3.21) & PA3 Fig.(3.22) \\
\hline PAE & $72 \%$ & $70 \%$ & $75.6 \%$ \\
\hline Power & $29 \mathrm{dBm}$ & $29 \mathrm{dBm}$ & $29.5 \mathrm{dBm}$ \\
\hline Gain & $18 \mathrm{~dB}$ & $17 \mathrm{~dB}$ & $16 \mathrm{~dB}$ \\
\hline AM-AM & $(0.2)-(1) \mathrm{dB} / \mathrm{dB}$ & $(0.1)-(0.6) \mathrm{dB} / \mathrm{dB}$ & $(0.2)-(1) \mathrm{dB} / \mathrm{dB}$ \\
\hline AM-FM & $(-0.2)-(0.6)$ degrees/dB & $(-0.2)-(1)$ degrees/dB & $(-0.2)-(0.2)$ degrees/dB \\
\hline $\mathrm{TH}$ & $<-35 d B c$ & $<-38 d B c$ & $<-46 d B c$ \\
\hline
\end{tabular}

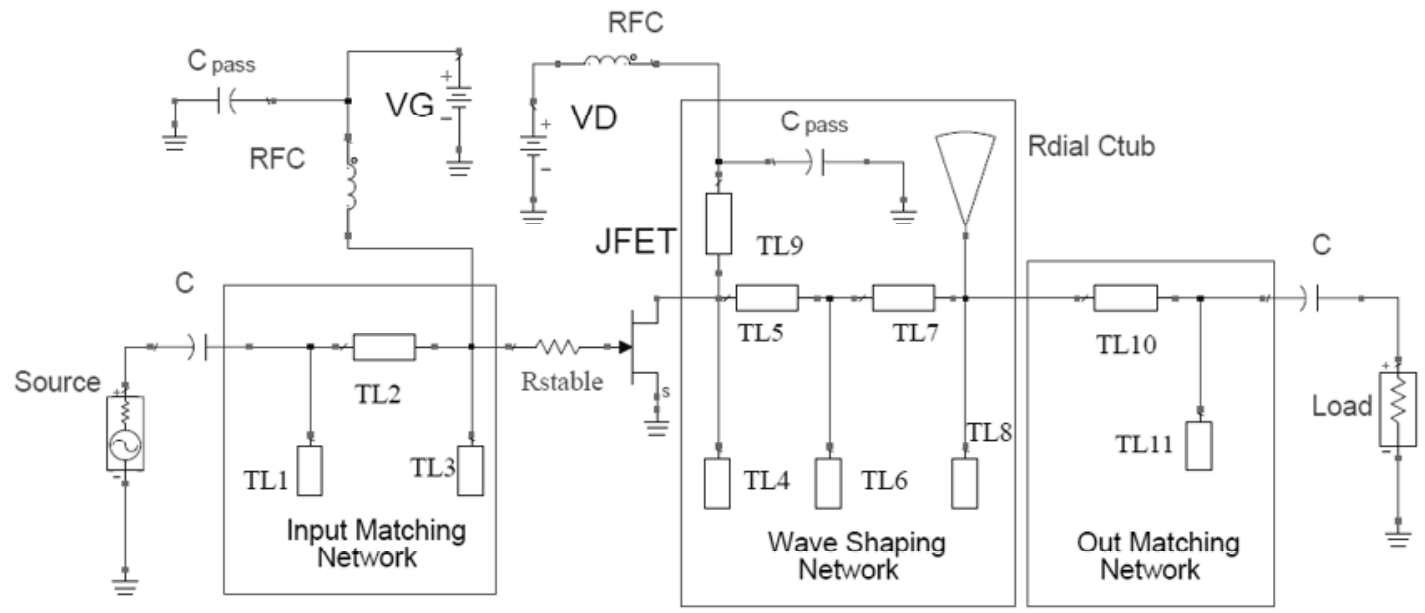

الشكل (8) دائرة مكبر القدرة نوع (F) الدائرة النهائية (PA3) 
5. ت تليل ومناقشة النتائج

تم در اسة وتحديد التوافقيات المؤثرة في تثكيل موجة الفولتية و التيار و حساب الكسب و الكفاءة ومقدار التشويه في

الزاوية والاتساع ومقدار التشويه في المركبة الثالثة نسبة إلى المركبة الأساسية (TH dBc)

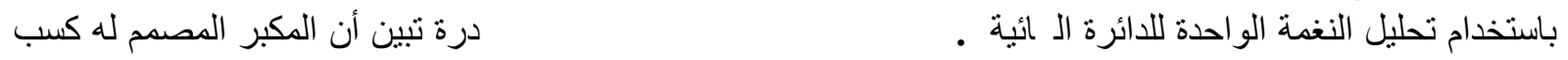

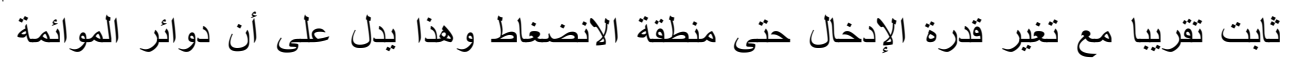

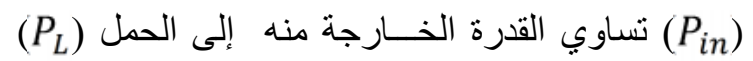

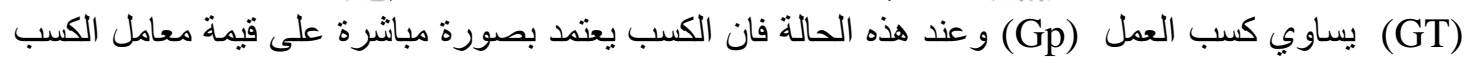

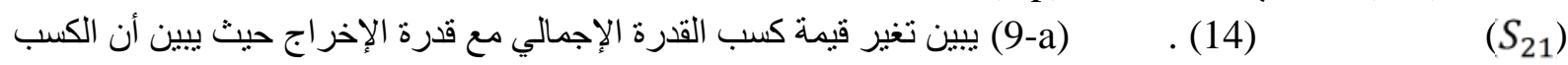

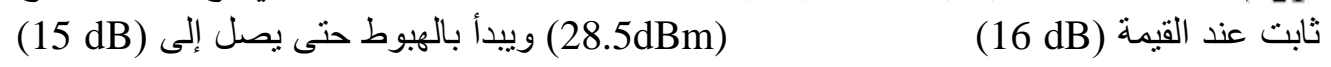

(29.91dBm) وهذا يعني أن المكبر يمتاز بكسب ثابت لمدى واسع من تغير القدرة الداخلة .

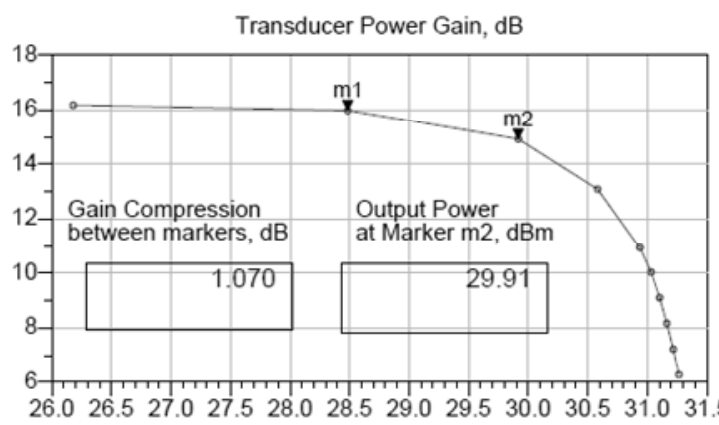

(a) Fund. Output Power, dBm

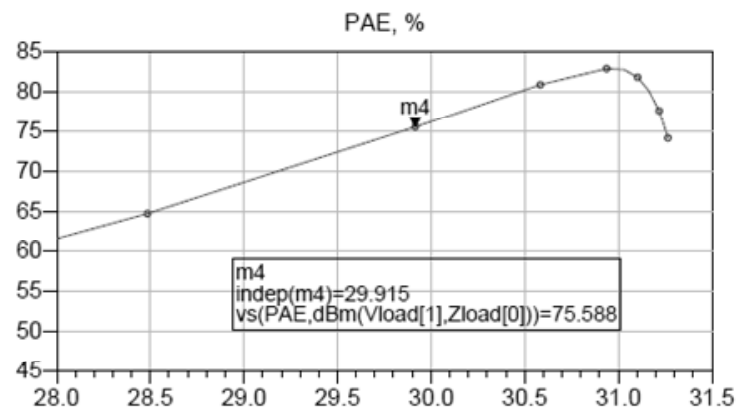

(b)

\section{الثكل (9) (a) تغيير كسب القدرة (b) تغيير كفاءة القدرة المضافة، مع قدرة الاخراج}

لقد كان لتصميم دائرة تشكيل الموجة التي تم من خلالها السيطرة على اتساع مركبات الفولتية والتيار المختلفة

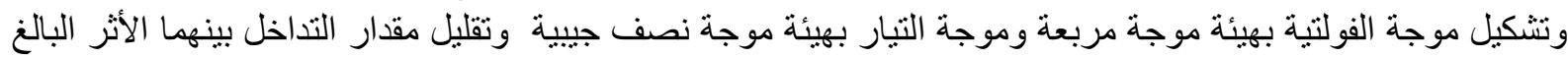

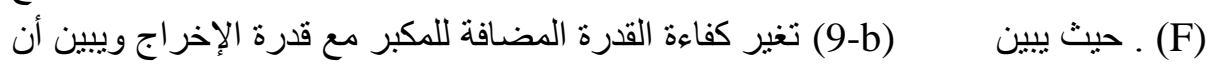

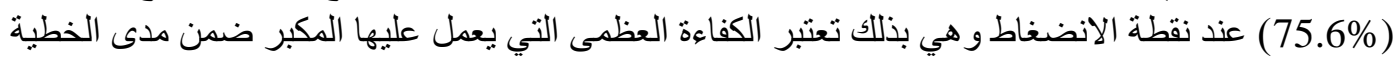

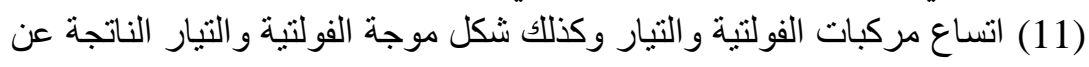
المناسبة . وبين

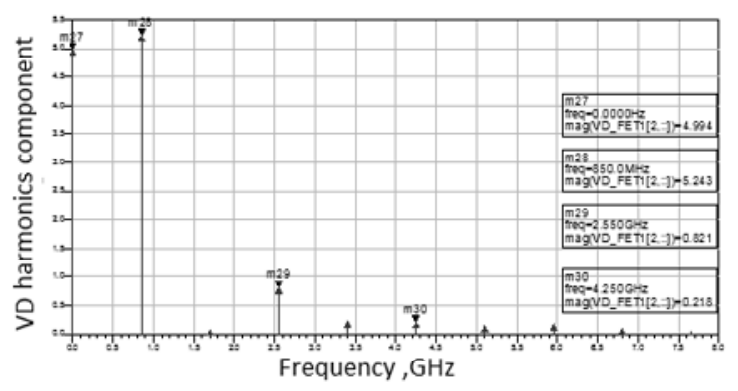

(a)

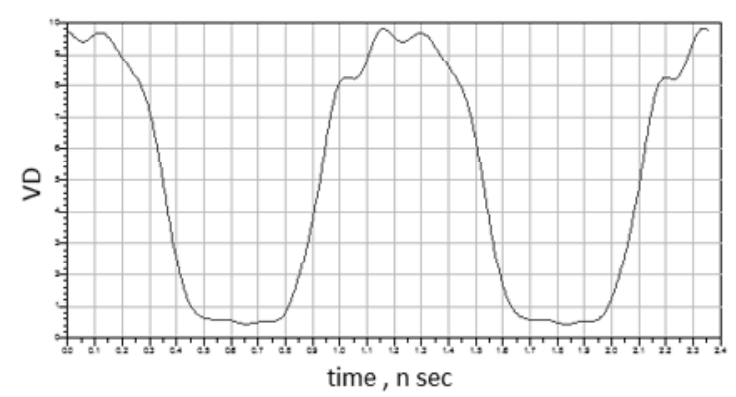

(b)

الشكل (10) مركبات فولتية المصرف والموجة الناتجة عنها 


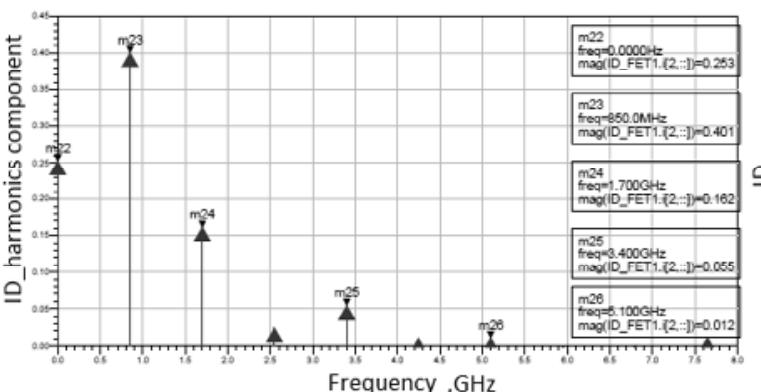

(a)

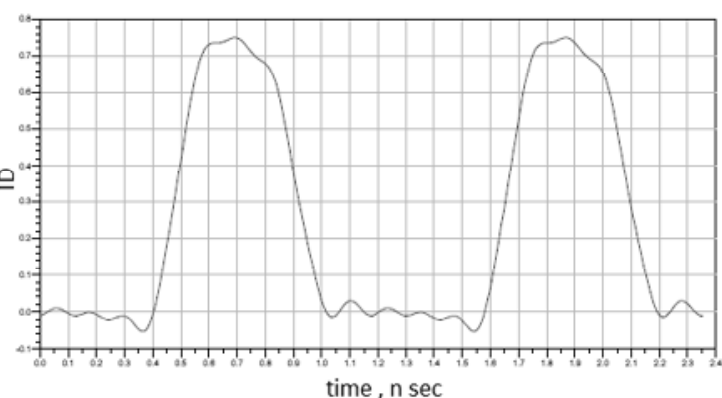

(b)

الشكل (11) مركبات تيار المصرف والموجة الناتجة عنها

فبحسب التصميم كانت المركبات الفردية الداخلة في تشكيل موجة الفولتية هي (1,3,5) و هذا مانجده في (10) حيث

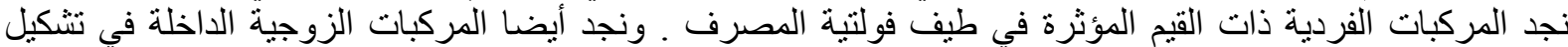

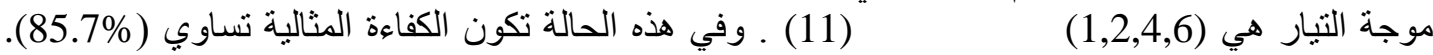

$P A E=\eta\left(1-\frac{1}{G T}\right)$

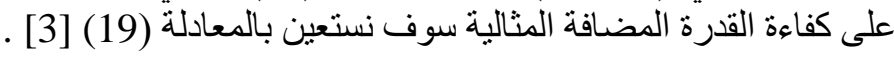
ومن تطبيق المعادلة السابقة على المكبر في منطقة الانضغاط (1dB) نحصل على قيمة القدرة المضافة المثالية و وذه النتيجة مقاربة للنتيجة التي تم الحصول عليها من نتائج المحاكاة مما يدل على عمل دائرة تشكيل الموجة بشكل جيد ـ و الفرق بين النتيجتين يعود إلى عدم السيطرة على اتساع مركبات الفولتية و التيار بشكل مثالي ولعدم إلغاء المركبات الزوجية من طيف إثارة الفولتية بصورة نهائية التئية وكذللك لم يتم إلغاء المركبات

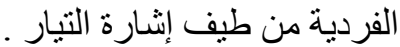
يعد مقدار التشويه في الزاوية والئار الاتساع ومقدار اتساع التو افقيات المتولدة نسبة إلى المركبة الأساسية من أهم مقاييس

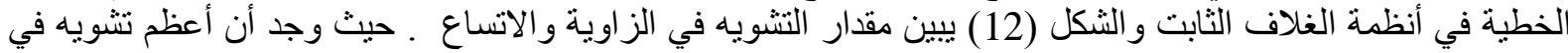

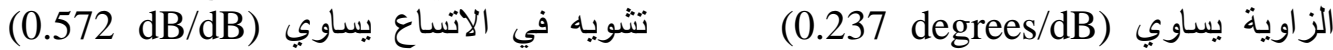

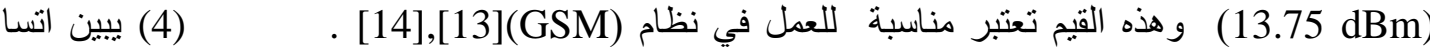
التو افقيات نسبة إلى المركبة الأساسية .
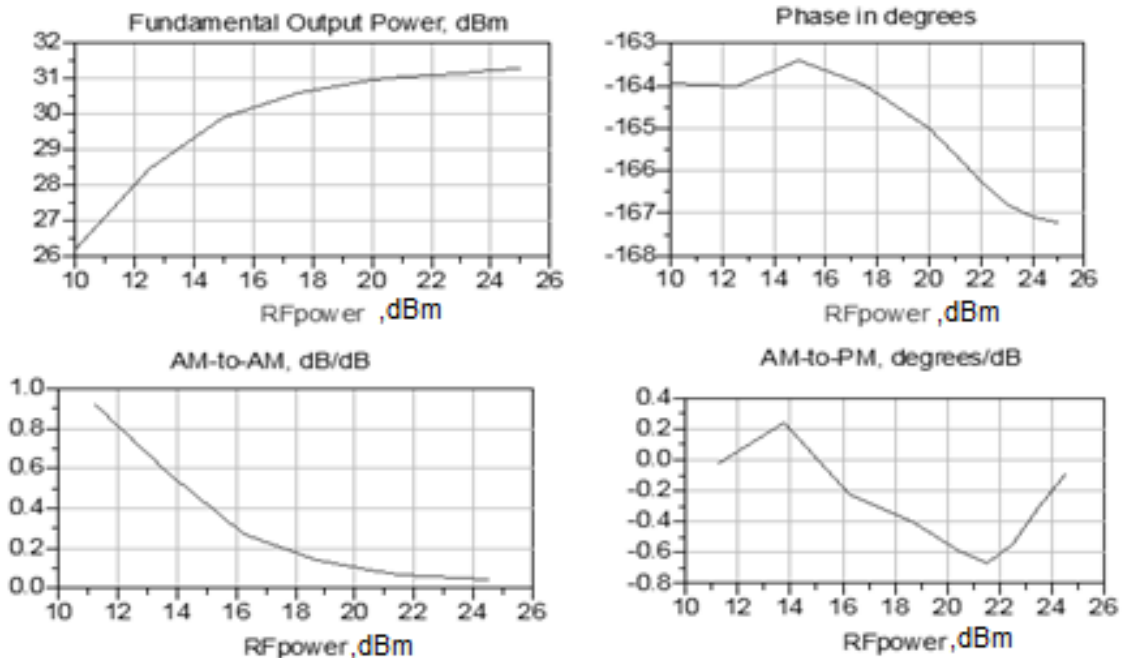

الشكل (12) يبين مقدار التشويه في الزاوية والاتساع 


\begin{tabular}{|c|c|c|c|c|c|}
\hline $\begin{array}{l}\text { Available } \\
\text { source } \\
\text { power dBm }\end{array}$ & $\begin{array}{c}\text { Fundamental } \\
\text { output power } \\
\text { dBm }\end{array}$ & $\begin{array}{c}\text { Second } \\
\text { harmonic } \\
\mathrm{dBc}\end{array}$ & $\begin{array}{c}\text { Third } \\
\text { harmonic } \\
\mathrm{dBc}\end{array}$ & $\begin{array}{c}\text { Fourth } \\
\text { harmonic } \\
\mathrm{dBc}\end{array}$ & $\begin{array}{c}\text { Fifth } \\
\text { harmonic } \\
\mathrm{dBc}\end{array}$ \\
\hline 10.00 & 26.18 & -63.79 & -55.61 & -41.41 & -68.3 \\
\hline 12.50 & 28.485 & -64.04 & -55.81 & -40.34 & -63.72 \\
\hline 15.00 & 29.915 & -64.31 & -46.31 & -38.93 & -63.79 \\
\hline
\end{tabular}

(5SM) بين النتائج التي تم الحصول عليها وبين نتائج مجمو عة من الباحثين الذين عملو ا في تطبيقات (-)

الجدول (5) مقارنة النتائج بالأعمال السابقة

\begin{tabular}{|c|c|c|c|c|c|c|}
\hline Ref. & PAE\% & $\begin{array}{c}\text { maximum output } \\
\text { power/ dBm }\end{array}$ & $\begin{array}{c}\text { Gain } \\
(\mathrm{dB})\end{array}$ & $\begin{array}{c}\text { Third } \\
\text { Harmonic dBc }\end{array}$ & $\begin{array}{c}\text { Amplifier } \\
\text { Type }\end{array}$ & $\begin{array}{c}\text { Transistor } \\
\text { TYPE }\end{array}$ \\
\hline$[5]$ & 88.97 & 38.35 & 13.35 & 63.241 & Class F & GaN \\
\hline$[10]$ & 74 & 20 & 9 & -42 & Class F & CaAs \\
\hline$[13]$ & 78.1 & 29 & 10.55 & -31 & Class E & CaAs \\
\hline$[14]$ & 62.8 & 36.6 & 6.9 & $\sim$ & Doherty & CaAs \\
\hline$[15]$ & 68.6 & 31.4 & 14.7 & -48.8 & Class F & CaAs \\
\hline $\begin{array}{c}\text { This } \\
\text { work }\end{array}$ & 75.6 & 29.5 & 15 & -46 & Class F & CaAs \\
\hline
\end{tabular}

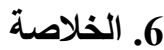

تم تصميم مكبر قدرة نوع (F) بطريقة الامثلية حيث من خلال هذا التصميم تم بناء مكبر قدرة له قدرة إخر اج عالية

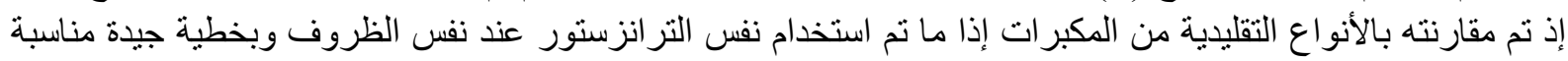

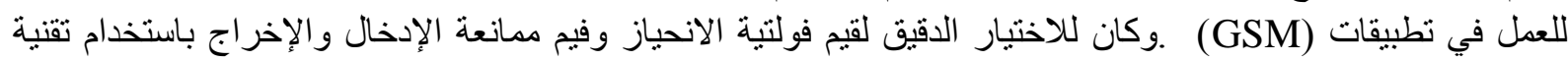

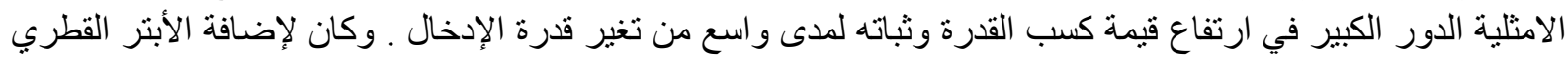

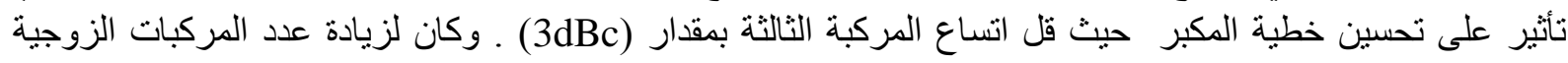

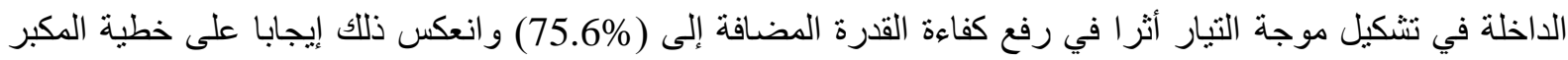

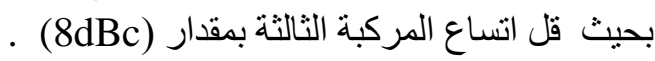

Reference

1. K. Chen, D. Peroulis, " Design of Highly Efficient Broad band Class-E Power Amplifier Using Synthesized Low-Pass Matching Networks",IEEE, Microwave Theory and Techniques, Vol. 59, No. 12, December 2011.

2. A . Z. Markos ,"Efficiency Enhancement of linear CaN RF Power Amplifier Using the Doherty Technique ", Ph.D , Thesis , electrical engineering /computer science. Kassel University,2008 .

3. Kyu Hwan An, " CMOS RF Power Amplifier for Mobial Wireless Communication", Ph.D Thesis, Georgia Institute of Technology ,December 2009.

4. Ockgoo Lee, "High Efficiency Switching CMOS Power Amplifier for Wireless Communications ",Ph.D. Thesis, Georgia Institute of Technology December 2009.

5. K. S. Tsang," Class-F Power Amplifier with Maximized PAE", M.Sc. Thesis, the Faculty of California Polytechnic State University, San Luis Obispo, August 2010.

6. Chris Bowick," RF Circuit Design"Newnes, 1982, P.66-70.

7. Guillermo, Gonzlaez "Microwave Transistor Amplifiers Analysis and Design", Prentice Hall, Second edition,1997,P.184-194. 
8. [8] I. A. Glover, S. R. Pennock and P. R. Shepherd, "Microwave Devices ,Circuits and Subsystem for Communication Engineering " John Wiley \& Sons Ltd, 2005 , P. 219-223.

9. J. Moon, J. Lee, R. S. Pengelly , R.Baker, and B. Kim, "Highly Efficient Saturated Power Amplifier ",IEEE Microwave Magazine, January/February 2012,P.125-131.

10. Shirt Fun OOI," Design of a high efficiency class f Power Amplifier integrated with Microstrip Antenna ", Ph.D. Thesis, school of computing ,engineering and information sciences northumbria university at newcastle ,2007.

11. A.Grebennikov ,N.O. Sokal , "Switchmode RF Power Amplifiers" Elsevier, 2007,P.95-106

12. M. Wren, T. J. Brazil, " Experimental Class-F Power Amplifier Design Using Computationally Efficient and Accurate Large-Signal pHEMT Model" IEEE transactions on microwave theory and techniques, VOL. 53, NO. 5, MAY 2005,P1723-1731.

13. W.K. Yaqoop ,"Improving The Performance of Class-E Power Amplifier in Mobile Unit of Cellular System ", Ph.D. Thesis, Electrical Engineering, University of Mosul, 2012.

14. k. H. Hamid," Study and Design of Doherty Power Amplifier" , M.Sc. Thesis, Electrical Engineering, University of Mosul , 2012.

15. R. B. Mohammed," Study and Design of Power Amplifiers for Cellular communications", M.Sc. Thesis, Electrical Engineering, University of Mosul , 2010.

تم اجراء البحث في كلية ألهندسة = جامعة ألموصل 